\title{
Determinación de la calidad físico-química de la miel de abeja comercializada en Quito y comparación con la miel artificial
}

\author{
(Determination of the physicochemical quality \\ of bee honey sold in Quito and comparison with artificial honey)
}

\author{
Doris Velásquez ${ }^{1}$, Lorena Goetschel ${ }^{2}$
}

\begin{abstract}
Resumen
Se evaluó la calidad de 27 muestras de miel de abeja que se comercializa en los mercados del sur de Quito mediante los análisis físico-químicos establecidos como requisitos en la Norma NTE INEN 1572-1988. Los resultados obtenidos se compararon con los mismos análisis realizados en una muestra de miel artificial. Los métodos utilizados para cada determinación analítica fueron: AOAC 962.37 para densidad, AOAC 962.19 para humedad, AOAC 52.729 para acidez, AOAC 945.79 para sólidos insolubles, AOAC 920.181 para cenizas, AOAC 980.23 para hidroximetilfurfural, AOAC 958.09 para diastasa y el método de Luff-Schoorl para la determinación de azúcares. Con excepción de una muestra que no cumplió el valor máximo del parámetro del contenido de hidroximetilfurfural, y tres muestras que no cumplieron con el porcentaje de azúcares reductores, toda la miel de abeja analizada cumplió con los requisitos de la norma, aunque presentaron resultados con diferencias significativas en varios parámetros entre las diferentes marcas. En cuanto a la comparación de la miel de abeja con la miel artificial existió diferencia significativa en el contenido de sólidos t en agua, azúcares reductores, sacarosa e hidroximetilfurfural, y la actividad de la diastasa.
\end{abstract}

\section{Palabras clave}

Miel de abeja; parámetros de calidad; Apis mellifera; análisis físico-químicos.

\begin{abstract}
The quality of 27 bee honey samples commercialized in the southern markets of Quito was evaluated by performing the physicochemical analyses required by the quality standard of the Ecuadorian Service for Standarization NTE INEN 1572-1988. The results were compared with the same type of analyses performed on a sample of artificial honey. The methods used for each analytical determination were: AOAC 962.37 for density, AOAC 962.19 for humidity, AOAC 52.729 for acidity, AOAC 945.79 for insoluble solids, AOAC 920.181 for ash, AOAC 980.23 for hydroxymethylfurfural, AOAC 958.09 for diastase and the Luff-Schoorl method for the determination of sugars. With the exception of one sample that did not comply the maximum value of the content parameter of hydroxymethylfurfural, and three samples that did not comply with the percentage of reducing sugars, all the samples of bee honey met the requirements of the national standard, although they showed results with significant differences in several parameters among different brands. As for the comparison between bee honey and artificial honey, there was a significant difference in the content of insoluble solids in water, reducing sugars, sucrose and hydroxymethylfurfural, and the activity of diastase.
\end{abstract}

\section{Keywords}

Bee honey; quality parameters; Apis mellifera; physicochemical analysis.

\section{Introducción}

La miel de abeja es consumida en todo el mundo como un sustituto del azúcar por su poder edulcorante, así como también por sus propiedades nutricionales y medicinales. De acuerdo con la Organización para la Alimentación y la Agricultura FAO, los principales países productores de miel son China y Estados Unidos, con una producción acumulada desde el año 2012 hasta el 
2016 de 2389760.00 TM y 357653.00 TM, respectivamente, mientras que la producción en Ecuador en el año 2012 fue de 856 TM (FAOSTAT, 2016).

El Ecuador por sus características climáticas y geográficas tiene gran potencial para la apicultura. Datos del Ministerio de Agricultura y Ganadería indican que en 2015 existían 912 explotaciones apícolas con 12188 colmenas catastradas, las que se calcula pueden llegar a ser 200000 colmenas (MAG, 2015), lo que incrementaría dieciséis veces la cantidad de colmenas y, por tanto, su producción.

El Codex Alimentarius define a la miel de abeja como "la sustancia dulce natural producida por abejas Apis mellifera a partir del néctar de las plantas o de secreciones de partes vivas de estas o de excreciones de insectos succionadores de plantas". Además indica que "la miel vendida como tal no deberá contener ningún ingrediente adicional, incluidos los aditivos alimentarios ni tampoco adición alguna que no sea miel" (CODEX STAN 12-2001). Esta norma internacional, así como la norma técnica ecuatoriana (NTE) INEN 1572 (INEN, 1988) fija los parámetros de calidad de la miel de abeja, la misma que fue actualizada posteriormente a este estudio (INEN, 2016).

La miel de abeja es un producto natural susceptible a la presencia de contaminantes. Los contaminantes más usuales que se encuentran son los de origen ambiental como: pesticidas, metales pesados, isotopos radioactivos, contaminantes orgánicos como los bifenilos policlorados (PCB's), bacterias patógenas y organismos genéticamente modificados. Además, se pueden encontrar contaminantes propios del producto como como acaricidas y antibióticos utilizados para el control de las enfermedades de estos insectos. La presencia de contaminantes químicos en este alimento puede afectar la salud de los seres humanos que lo consumen, esto depende de la sustancia, su concentración y frecuencia de consumo, también puede provocar efectos inmediatos como alergias o reacciones cutáneas, y a largo plazo efectos teratogénicos, mutagénicos y carcinogénicos. La presencia de pesticidas y otros contaminantes ha causado también la disminución del número de insectos a nivel mundial, mientras que el uso extendido de antibióticos ha incrementado la resistencia antimicrobiana (Noori Al-Waili, 2012).

Los microorganismos presentes en la miel pueden ser bacterias, mohos y levaduras que provienen de las mismas abejas, del medio ambiente o de su procesamiento. La presencia de esporas de Clostridium botulínico en la miel ha sido objeto de varios estudios, ya que constituye un grave peligro para los infantes porque puede causar el botulismo infantil, que se produce cuando las esporas se reproducen en el tracto digestivo y producen la toxina botulínica que ocasiona fatales consecuencias, especialmente, en recién nacidos e infantes. Los estudios realizados en países como Argentina, Brazil, Japón, Finlandia y Estados Unidos evidencian la presencia de esporas de este microorganismo en algunas muestras (Noori Al-Waili, 2012) de producto.

La calidad y la inocuidad de la miel de abeja comercializada en Ecuador requiere ser investigada y solamente escasos estudios formales se han reportado en este país.Un estudio importante es el realizado recientemente en 25 muestras comerciales, obtenidas en Pichincha y Loja (Salvador, 2019), donde se concluye que la existencia de poca información sobre este alimento muestra la necesidad de investigar sobre el tema. El presente estudio permite contar con datos sobre la calidad de la miel que se comercializa en Quito con base en el cumplimiento de los parámetros fisicoquímicos exigidos por la NTE INEN 1572:1988; además, cuenta con datos sobre los parámetros dentro de la normativa que presentan diferentes valores para la miel de abeja y miel artificial. 


\section{Metodología}

Este estudio se realizó en dos fases; la primera consistió en cuantificar las marcas de miel de abeja comercializadas en los mercados del sur de Quito, para ello se elaboró y aplicó una encuesta a los vendedores que se encontraban en los sitios de expendio. Una vez determinadas las marcas de mayor frecuencia de comercialización se realizó un muestreo aleatorio, y se tomaron muestras de cada marca.

La segunda fase se basó en realizar los análisis fisicoquímicos de las muestras de miel de abeja y de una miel artificial por triplicado, según los parámetros establecidos en la normativa ecuatoriana de referencia, para establecer el cumplimiento o incumplimiento de esta. Posteriormente, se compararon los resultados de cada parámetro entre las muestras de miel de abeja y la miel artificial. Los métodos utilizados para cada análisis fueron los métodos de la Association of Oficial Analytical Chemists (AOAC, 1984): AOAC 962.37 para densidad, AOAC 969.38B para humedad, AOAC 962.19 para acidez, AOAC 945.79 para sólidos insolubles, AOAC 920.181 para cenizas, AOAC 980.23 para hidroximetilfurfural, AOAC 958.09 para diastasa y para azúcares el método de Luff-Schoorl.

Esta investigación cuantitativa exploratoria experimental se llevó a cabo en los laboratorios de: Análisis de Alimentos, Análisis Instrumental y OSP del Área de Alimentos de la Facultad de Ciencias Químicas de la Universidad Central del Ecuador.

La población corresponde a los productos apícolas de secreción declarados en forma de miel de abeja, que se expenden en los mercados del sur de Quito. La muestra se obtuvo por medio de un muestreo aleatorio. La cantidad de muestra tomada es proporcional al volumen de venta de cada marca de miel de abeja (de acuerdo con la encuesta). Cada muestra fue sometida a los análisis físicos y químicos que se describen a continuación:

2.1 Densidad. Se analizó mediante el método AOAC 962.37 detallado en la NTE INEN 1632 (INEN, 2012) que se basa en la determinación del peso del agua y de la muestra de un volumen constante, manteniendo el picnómetro en un baño térmico a $27^{\circ} \mathrm{C}$.

2.2 Humedad. Para su determinación se utilizó el método AOAC 969.38B que consiste en medir el índice de refracción de la muestra y obtener el valor mediante el uso de la tabla, que está detallada en la norma INEN 1632 (INEN, 2012).

2.3 Acidez. Se basa en la titulación por retroceso con solución de hidróxido de sodio y ácido clorhídrico $0.05 \mathrm{~N}$ de acuerdo con el método oficial AOAC 962.19 descrito en la NTE INEN 1634 (INEN, 1989).

2.4 Contenido de azúcares. Se realizó mediante el método de Luff-Schoorl (Kirk, 1999), que consiste en añadir solución de Luff a la muestra diluida, llevar a reflujo, colocar ioduro de potasio y ácido sulfúrico y titular con solución de tiosulfato de sodio hasta obtener una coloración café clara, añadir solución de almidón y continuar con la titulación hasta color blanco, a la par se realiza un ensayo en blanco. Para determinar azúcares totales se realiza el mismo procedimiento, pero la muestra previamente se somete a hidrólisis, mediante la adición de ácido clorhídrico concentrado en la disolución de miel y se mezcla en baño hasta que la solución presente el primer cambio de coloración (color más intenso). Se neutraliza con hidróxido de sodio hasta que el color rosado persista.

2.5 Sólidos insolubles. Se utilizó el método oficial AOAC 945.79, detallado en la NTE INEN 1635 (INEN, 2012), que consiste en determinar la cantidad de sólidos que no se disuelven y quedan en el papel filtro cuando la muestra en solución es filtrada.

2.6 Cenizas. Se basó en el método oficial AOAC 920.181 que consiste en determinar el peso del residuo seco de la muestra, calcinado en la mufla a $600{ }^{\circ} \mathrm{C}$ hasta peso constante y presencia de cenizas blancas, según se describe en la NTE INEN 1636 (INEN, 1989). 
2.7 Hidroximetilfurfural (HMF). Se aplicó el método oficial AOAC 980.23 detallado en la NTE INEN 1637 (INEN, 2012) que es un método espectrofotométrico que consiste en disolver la muestra en agua destilada, añadir solución de ferrocianuro de potasio y solución de acetato de zinc, y llevar hasta un volumen fijo con agua destilada. Se mide la absorbancia a 284 y 336 nm de agua y de la solución de referencia (solución bisulfato de sodio), y se obtiene el resultado mediante la fórmula de cálculo.

2.8 Número de diastasa. Para su determinación se utilizó el método oficial AOAC 958.09; es una técnica analítica espectrofotométrica que se basa en pesar la muestra y disolver en agua destilada y añadir solución tampón, luego se transfiere a un matraz que contiene un volumen de solución de cloruro de sodio y se afora. Se coloca en baño térmico junto con una solución de almidón por 15 minutos. Luego se agrega la solución de almidón en la de miel, se agita, se deja reposar y luego de cinco minutos se toma $1 \mathrm{ml}$ con una pipeta y se agrega a $10 \mathrm{ml}$ de solución diluida de yodo, se mezcla y se mide en el espectrofotómetro a $660 \mathrm{~nm}$.

2.9 Análisis estadístico. Se utilizó un análisis de varianza (ADEVA) de un factor para determinar si existe diferencia entre las muestras y para comparar los resultados obtenidos entre las muestras y la miel artificial, se aplicó una prueba 〈t〉, donde se comparan los valores de las medias de las tres marcas más comercializadas, con los valores de miel artificial. La prueba de hipótesis que se aplicó es que los resultados de los análisis físicos y químicos de la miel de abeja de las marcas 1, 2 y 3 son diferentes a los resultados de la miel artificial.

\section{Resultados}

3.1 Encuesta. La encuesta realizada a los vendedores en los sitios de expendio mostró que se encuentran 35 secciones en los nueve mercados del sur de Quito donde se comercializa miel de abeja, las que se distribuyen en cada mercado según la siguiente tabla:

Tabla 1. Distribución de las secciones destinadas al expendio de miel de abeja

\begin{tabular}{|l|l|l|l|l|l|l|l|l|l|l|}
\hline \# Mercado & 1 & 2 & 3 & 4 & 5 & 6 & 7 & 8 & 9 & Total: 9 mercados \\
\hline \# De secciones & 4 & 3 & 3 & 4 & 6 & 3 & 4 & 3 & 5 & Total: 35 secciones \\
\hline
\end{tabular}

La encuesta aplicada en todas las secciones permitió establecer que se comercializan cinco marcas de miel de abeja en estos sitios. Cada marca pertenece a diferente proveedor y se encuentran en diferente número de lugares de expendio: marca 1 en el $68.6 \%$, marca 2 en el 40.0 \%, marca 3 en el $31.4 \%$, marca 4 abarca el $14.3 \%$ y en un $8.6 \%$ se ubica la marca 5.

Tabla 2. Presencia y volumen de venta de cada marca en los lugares encuestados

\begin{tabular}{|c|c|c|c|c|c|}
\hline Marca & $\begin{array}{c}\text { Registro } \\
\text { Sanitario }\end{array}$ & $\begin{array}{c}\text { Número de locales que } \\
\text { expenden esta marca }\end{array}$ & $\begin{array}{c}\% \text { de presen- } \\
\text { cia de la marca }\end{array}$ & $\begin{array}{c}\text { Volumen de venta } \\
\text { (envases/semana) }\end{array}$ & $\begin{array}{c}\text { Código de } \\
\text { la muestra }\end{array}$ \\
\hline 1 & Sí & 24 & 68.6 & 196 & A, B y C \\
\hline 2 & No & 14 & 40.0 & 117 & D y E \\
\hline 3 & Sí & 11 & 31.4 & 81 & F y G \\
\hline 4 & Sí & 5 & 14.3 & 32 & H \\
\hline 5 & No & 3 & 8.6 & 15 & I \\
\hline
\end{tabular}


Dos de las cinco marcas (marca 2 y marca 5) no poseen registro sanitario, aunque sí cuentan con una etiqueta de identificación del producto. En un solo lugar de expendio se pueden encontrar más de una marca comercial. En la encuesta también se preguntó la frecuencia de compra y el $100 \%$ indicó que compra mensualmente al proveedor.

3.2 Muestreo. Con la información obtenida en la encuesta se realizó un muestreo no probabilístico a juicio, tomando en cuenta la distribución de los proveedores de miel de abeja y el volumen de venta para cada uno. Al ser la marca 1 la de mayor volumen de venta se muestreó en tres secciones en diferentes mercados, las marcas 2 y 3 en dos secciones, marcas 4 y 5 en una sección, respectivamente. Escogiéndose en total nueve sitios para el muestreo.

Se identificó con letras del alfabeto (A-G) a cada muestra que corresponde por cada marca que varió de acuerdo con el lugar dónde se tomaron las muestras, como lo indica la Tabla 2. Por ejemplo, a la marca 1 le corresponden las muestras identificadas con las letras $A$, B y C, que fueron tomadas en un mercado diferente cada una, al igual que las demás muestras que se detallan a continuación. Las muestras $\mathrm{D}$ y E corresponden a la marca 2, las F y $\mathrm{G}$ a la marca 3 , a la marca 4 le corresponde la letra $\mathrm{H}$ y, finalmente, la letra I para la marca 5 . Es decir que cada muestra fue tomada en diferentes mercados.

En cada lugar escogido se muestrearon tres envases de miel de abeja de la misma marca y lote de producción, las que se identificaron como A1, A2 y A3 para la muestra y mercado A, y así respectivamente para todas las muestras, las que fueron sometidas a los diferentes análisis físicos y químicos por triplicado.

Se adquirió un envase de miel artificial en un supermercado. Este producto se identifica en la etiqueta como miel de sacarosa y los ingredientes declarados son azúcar, agua, ácido cítrico, hojas de eucalipto y sorbato de potasio, cuyo aspecto, color y aroma es muy similar a la miel de abeja.

\subsection{Análisis fisicoquímicos}

Los promedios de los resultados de los análisis de las tres muestras de cada sitio de muestreo se indican en la Tabla 3, donde se las especificaciones de la norma, así como los resultados del análisis estadístico.

Los resultados muestran que de las 27 muestras analizadas, el $100 \%$ cumplen las especificaciones de la norma 1572:2008 para miel de abeja clase I, para los parámetros de humedad, acidez, porcentaje de sólidos insolubles, contenido de cenizas, porcentaje de sacarosa, contenido de HMF y número de diastasa. Existe una muestra que no cumple el parámetro de densidad, por lo que el 96 \% cumplen con el parámetro, mientras que tres muestras no cumplen el porcentaje de azúcares reductores, es decir, que el 92.5 \% cumplen con este parámetro.

Los resultados de los parámetros: densidad, humedad, acidez, sólidos insolubles, cenizas y sacarosa, no presentaron diferencias significativas entre las muestras analizadas, por lo que, a pesar de pertenecer a diferente marca (proveedor) y lugar de muestreo (mercado) su comportamiento fue similar.

Con respecto al contenido de azúcares reductores, sí existe diferencia significativa entre los mercados seleccionados, los valores obtenidos oscilan entre 65-75 \%. La prueba DMS agrupa a las muestras en cuatro rangos; en el rango (a) se ubican las muestras $\mathrm{E}$, D; el rango (b) lo conforman las muestras B, A, C; en el rango (c), se encuentran las muestras H, I, F y, por último, el rango (d) abarca las muestras I, F, G; en cada rango las muestras son estadísticamente iguales. 
Tabla 3. Resultados físico -químicos

\begin{tabular}{|c|c|c|c|c|c|c|c|c|c|c|}
\hline  & 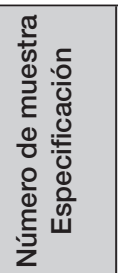 & 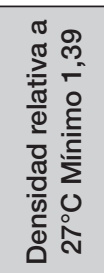 & 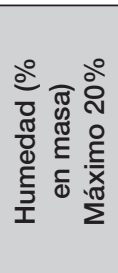 & 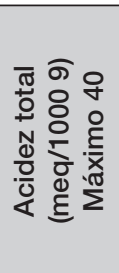 & 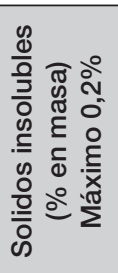 & 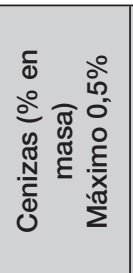 & 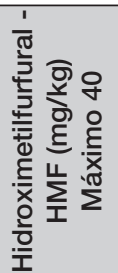 & 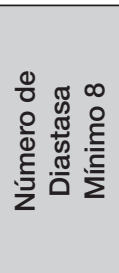 & 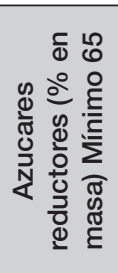 & 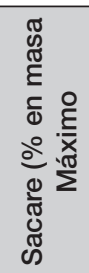 \\
\hline \multirow{4}{*}{ A } & A7 & 1,43 & 77,60 & 25,98 & 0,10 & 0,12 & 4,01 & 15,97 & 72,90 & 2,93 \\
\hline & $A 2$ & 1,47 & 16,00 & 28,07 & 0,07 & 0,23 & 4,24 & 16,44 & 71,60 & 2,71 \\
\hline & A3 & 1,43 & 16,20 & 27,31 & 0,07 & 0,20 & 5,17 & 14,73 & 72,95 & 2,23 \\
\hline & $\sim X$ & 1,44 & 16,60 & 27,12 & 0,08 & 0,18 & 4,47 & 15,71 & 72,48 & 2,62 \\
\hline \multirow{4}{*}{ B } & B1 & 1,46 & 17,72 & 28,05 & 0,09 & 0,23 & 2,25 & 15,76 & 72,95 & 2,49 \\
\hline & B2 & 1,40 & 16,12 & 27,50 & 0,06 & 0,22 & 3,56 & 15,40 & 72,81 & 2,93 \\
\hline & B3 & 1,43 & 17,28 & 26,67 & 0,06 & 0,22 & 3,23 & 15,20 & 72,56 & 2,09 \\
\hline & $\sim X$ & 1,43 & 77,04 & 27,41 & 0,07 & 0,22 & 3,01 & 15,45 & 72,77 & 2,50 \\
\hline \multirow{4}{*}{ C } & C1 & 1,47 & 16,12 & 27,40 & 0,09 & 0,13 & 4,14 & 15,35 & 71,96 & 2,45 \\
\hline & $\mathrm{C} 2$ & 1,39 & 16,16 & 28,11 & 0,07 & 0,23 & 4,09 & 16,25 & 72,13 & 2,92 \\
\hline & C3 & 1,42 & 16,24 & 28,24 & 0,06 & 0,19 & 4,75 & 15,48 & 73,08 & 2,10 \\
\hline & $\sim x$ & 1,43 & 16,17 & 27,92 & 0,08 & 0,19 & 4,33 & 15,69 & 72,39 & 2,49 \\
\hline \multirow{4}{*}{$D$} & D1 & 1,46 & 16,20 & 29,17 & 0,09 & 0,15 & 14,19 & 13,99 & 74,85 & 2,69 \\
\hline & $D 2$ & 1,45 & 16,00 & 27,01 & 0,07 & 0,18 & 17,41 & 14,08 & 74,89 & 2,03 \\
\hline & D3 & 1,44 & 16,60 & 29,12 & 0,08 & 0,23 & 71,21 & 13,27 & 75,87 & 2,70 \\
\hline & $\sim X$ & 1,45 & 16,27 & 28,43 & 0,08 & 0,19 & 14,27 & 13,78 & 75,20 & 2,48 \\
\hline \multirow{4}{*}{$E$} & $E 1$ & 1,42 & 16,28 & 27,39 & 0,04 & 0,23 & 13,18 & 13,30 & 76,09 & 2,07 \\
\hline & E2 & 1,44 & 16,96 & 26,51 & 0,11 & 0,22 & 14,97 & 13,49 & 75,38 & 3,03 \\
\hline & E3 & 7,47 & 16,36 & 26,58 & 0,11 & 0,29 & 15,03 & 14,00 & 75,51 & 2,30 \\
\hline & $\sim X$ & 1,44 & 16,53 & 26,83 & 0,09 & 0,25 & 14,39 & 13,60 & 75,66 & 2,47 \\
\hline \multirow{4}{*}{$F$} & $F 1$ & 1,41 & 18,00 & 26,87 & 0,06 & 0,07 & 19,80 & 12,44 & 65,75 & 2,77 \\
\hline & $F 2$ & 1,43 & 16,96 & 28,49 & 0,06 & 0,04 & 15,12 & 13,30 & 64,39 & 3,19 \\
\hline & F3 & 1,39 & 16,80 & 28,31 & 0,04 & 0,19 & 18,24 & 12,55 & 65,69 & 2,49 \\
\hline & $\sim X$ & 1,41 & 77,25 & 27,89 & 0,05 & 0,10 & 77,72 & 72,77 & 65,28 & 2,82 \\
\hline \multirow{4}{*}{ G } & G1 & 1,35 & 16,48 & 29,59 & 0,06 & 0,08 & 18,43 & 13,60 & 66,79 & 2,54 \\
\hline & G2 & 1,45 & 17,00 & 27,49 & 0,03 & 0,22 & 11,54 & 13,25 & 64,08 & 3,05 \\
\hline & G3 & 1,43 & 16,12 & 26,55 & 0,08 & 0,17 & 15,56 & 13,31 & 64,19 & 2,76 \\
\hline & $\sim X$ & 1,41 & 16,53 & 28,02 & 0,06 & 0,16 & 15,18 & 13,38 & 65,02 & 2,78 \\
\hline \multirow{4}{*}{ H } & $\mathrm{H1}$ & 1,46 & 16,80 & 29,03 & 0,06 & 0,08 & 18,66 & $\begin{array}{lll}11,41 \\
\end{array}$ & 65,41 & 3,12 \\
\hline & $\mathrm{H} 2$ & 7,42 & 77,24 & 26,79 & 0,03 & 0,14 & 18,77 & 11,04 & 66,63 & 2,31 \\
\hline & H3 & 1,43 & 16,80 & 29,52 & 0,02 & 0,26 & 18,50 & 11,14 & 65,33 & 3,23 \\
\hline & $\sim X$ & 1,44 & 16,95 & 28,44 & 0,04 & 0,16 & 18,64 & 11,20 & 65,79 & 2,89 \\
\hline \multirow{4}{*}{1} & 11 & 1,43 & 17,20 & 27,85 & 0,07 & 0,15 & 18,91 & 11,22 & 66,09 & 2,43 \\
\hline & 12 & 7,45 & 77,00 & 28,19 & 0,04 & 0,20 & 16,71 & 11,83 & 66,91 & 7,45 \\
\hline & 13 & 7,44 & 16,80 & 28,76 & 0,07 & 0,17 & 77,76 & 11,95 & 66,52 & 2,17 \\
\hline & $\sim X$ & 1,44 & 17,00 & 28,27 & 0,06 & 0,17 & 17,79 & 11,67 & 66,51 & 2,02 \\
\hline \multicolumn{2}{|l|}{ F calc. } & $0,75 n \mathrm{~s}$ & $1,55 n s$ & $1,00 \mathrm{~ns}$ & $1,89 n \mathrm{~ns}$ & $1,58 \mathrm{~ns}$ & $36,09^{*}$ & $40,67^{*}$ & $102,74^{*}$ & $1,16 n \mathrm{~s}$ \\
\hline \multicolumn{2}{|c|}{$\frac{\text { Valor Mínimo }}{\text { Valor Máximo }}$} & 1,35 & 16 & 25,98 & 0,02 & 0,04 & 2,25 & 11,04 & 64,08 & 1,45 \\
\hline \multicolumn{2}{|c|}{$\begin{array}{l}\text { Valor Máximo } \\
\# \text { mastros fuera de }\end{array}$} & 1,47 & 18 & 29,17 & 0,11 & 0,29 & 18,91 & 16,44 & 76,09 & 3,23 \\
\hline \multicolumn{2}{|c|}{$\begin{array}{l}\text { \# muestras fuera de la } \\
\text { norma }\end{array}$} & 1 & 0 & 0 & 0 & 0 & 0 & 0 & 2 & 0 \\
\hline \multicolumn{2}{|c|}{ Miel artificial } & 1,43 & 16,27 & 30,98 & 0,02 & 0,03 & & & 6,04 & 84,5 \\
\hline
\end{tabular}

* Especificaciones para miel de abeja-Clase I (Norma INEN NTE 1572 1988-04)

Ns-no existe diferencia significativa para ese parámetro considerando una F tabulada al 0.05 de 2.51 con un nivel de confianza del $95 \%$ no existe diferencia significativa.

** si existe diferencia significativa entre los resultados del análisis de este parámetro.

*** Muestras fuera de la norma: Densidad.-muestra G: 1,35 g/cc; \% de azúcares reductores.-muestra F2: 64.39 \% y dos muestras G: G2: $64.08 \%$ y G3:64.19\%. 
En el caso del parámetro HMF los resultados obtenidos van de 3.01 a 18.64 mg/kg, la diferencia detectada entre las muestras es significativa, la prueba DMS agrupa a las muestras en cuatro rangos; el primer rango está conformado por las muestras $H_{1} I$, F, que son estadísticamente iguales, lo mismo ocurre con el rango (b) que contiene a las muestras I, F, G, en el rango (c) se ubican las muestras G, E, D siendo iguales entre si y, finalmente, en el rango (d) se agrupan las muestras $A, C, B$, que también son diferentes al resto de rangos.

El número de diastasa se encuentra entre 11.20 y 15.71, la diferencia encontrada entre los mercados seleccionados sí es significativa, la prueba DMS clasifica a los tratamientos en cuatro rangos; en el rango (a) se agrupan los tratamientos A, C, B que son iguales entre sí, de igual forma, el segundo rango que lo conforman $D, E$, G, para el rango (c) se juntan $G$, F, siendo estadísticamente iguales y en el último rango se encuentran I, H, que son similares entre sí.

De acuerdo con la prueba (t), las medias de densidad y humedad de miel artificial son iguales a las que presenta el proveedor 1. Sin embargo, en cuanto a la acidez, sólidos insolubles, cenizas y sacarosa, su contenido es diferente. Con respecto al proveedor 2, la prueba (t) determina que la densidad, humedad y acidez son iguales a los de miel artificial, mientras que el contenido de sólidos insolubles, cenizas y sacarosa, difieren, estableciéndose que estas propiedades no son iguales. Finalmente, para el proveedor 3, se detecta que tanto la densidad, humedad, acidez y cenizas, no son diferentes a la miel artificial, los que si difieren son el contenido de cenizas y sacarosa.

Se evidencia que la miel artificial presenta un contenido de sacarosa de $84.5 \%$, mientras que la miel de abeja no supera el $3 \%$; los sólidos insolubles que presenta la miel de abeja al igual que el contenido de cenizas son elevados con respecto a la miel artificial, y el contenido de azúcares reductores de la miel artificial es muy bajo (6.04 \%) en relación con los resultados obtenidos en la miel de abeja que contiene más del $65 \%$. En la miel artificial no se puede identificar la presencia de HMF y el análisis del número de diastasa muestra un resultado negativo.

\section{Discusión}

En este caso el 60 \% de las marcas analizadas (tres de cinco) tuvieron registro sanitario. El registro sanitario a partir de 2015 cambió por la notificación sanitaria, cuya obtención es obligatoria para los alimentos procesados. Según el artículo 14 de la Resolución ARCSA-DE067-2015-GGG, la miel de abeja consta como uno de los alimentos que se exceptúa del cumplimiento de la obtención de Notificación Sanitaria, pero está sujeta a la vigilancia y control sanitario por parte de la Agencia Nacional de Regulación, Control y Vigilancia Sanitaria-ARCSA, en coordinación con las entidades correspondientes. El mencionado artículo indica en su literal b) sobre excepciones: "Productos alimenticios de origen animal sean estos crudos, refrigerados o congelados, incluyendo productos cortados; siempre y cuando no hubiesen sido sometidos a procesos tecnológicos de transformación, modificación y conservación. Se incluye huevos en estado natural y miel de abeja" (ARCSA - MSP, 2015).

Los resultados de este estudio señalan un alto nivel de cumplimiento de las muestras analizadas, con relación a los parámetros de la normativa nacional e internacional, de manera similar a lo obtenido por Wilmer Martínez (Martínez, 2009) quien realizó el análisis fisicoquímico de la miel de abeja producida artesanalmente en Ecuador y de la de origen extranjero, basándose en los parámetros de calidad que establece la NTE INEN 1572:2008, en el que resultó que todas las muestras analizadas cumplieron los parámetros establecidos en este documento 
normativo y que únicamente se presentaron pequeñas variaciones entre los resultados analíticos de la miel artesanal e industrial.

Los análisis de 30 muestras de miel de abeja de diferentes sitios de comercialización en Cuenca realizados en la Universidad del Azuay (Morales, 2016) indican que únicamente 11 de las 30 tienen registro sanitario, y en lo referente al cumplimiento con la NTE INEN 1572:1988 (INEN, 1988) el 100 \% cumplen con el parámetro de densidad, cenizas y sólidos insolubles. En cuanto al producto que no cumple con los parámetros establecidos: el 76.7 \% no cumple el parámetro de diastasa, el 36.7 \% tampoco cumple el parámetro de HMF, el 3.3 \% no cumple el parámetro de azúcares reductores, el 20 \% no cumple el parámetro de sacarosa, el 6,7\% no cumple con la humedad y el 13,3\% no cumple con el límite de acidez. Al revisar el presente estudio con el realizado por la Universidad del Azuay, se observa similitud en cuanto al cumplimiento de las especificaciones de densidad, cenizas y sólidos insolubles. Sin embargo, las muestras analizadas en Cuenca presentan niveles de incumplimiento superiores en los demás parámetros. Igualmente, en los dos estudios se evidencia la presencia de productos con y sin registro sanitario.

En el estudio "Authenticity of Ecuadorian commercial honeys" (Vit, Chievano, Zuccato, y Finotello, 2015) realizado en la Universidad de Machala, 34 de las 42 muestras analizadas provenientes de cinco provincias del Ecuador son genuinas, según los análisis realizados por medio del método de dietil éter, confirmados luego con espectrometría HNMR que permite el análisis del producto diluido en agua deuterada. De acuerdo con este estudio los parámetros indicativos de la presencia de adulteración son: la ausencia de aminoácidos, el elevado nivel de HMF, la presencia de ácido cítrico como evidencia de la manufactura, bajos niveles de azúcares propios de la miel, y el nivel elevado de sacarosa. Estos resultados son similares a los del presente estudio en cuanto a mostrar que la miel artificial cuenta con bajos niveles de azúcares reductores y un elevado nivel de sacarosa. No coincide la determinación de HMF que no se detectó en la miel artificial. Cabe destacar el uso del método de dietil éter que es una técnica muy sencilla que permite identificar fácilmente la miel adulterada.

El estudio físico químico y microbiológico realizado a siete muestras de miel de Apis mellifera en Antioquia Colombia (Velásquez Giraldo A.M., 2013) reveló también que todos los productos analizados cumplen la Resolución 1057 (INVIMA, 2010) y la norma CODEX Stan 12-1981. En ese estudio se realizaron los análisis de densidad, humedad y contenido de azúcares (sacarosa, glucosa y fructosa) que se incluyen en la norma CODEX, y otros como actividad de agua, $\mathrm{pH}$, acidez libre $(\mathrm{meq} / \mathrm{Kg})$, índice de refracción, rotación específica y análisis microbiológicos que no se mencionan en la norma CODEX, pero sí en la norma colombiana.

Cabe mencionar que los parámetros en los que se obtienen resultados fuera de los parámetros de la norma para la miel artificial no necesariamente son indicadores de adulteración. Así el HMF es un indicativo de la frescura y calentamiento de la miel, además que la disminución de la actividad diastasa también es un indicativo del sobrecalentamiento (White, 1994), por lo que estos parámetros no pueden ser considerados como indicativos de adulteración. El parámetro de contenido de sólidos insolubles es un indicativo de control higiénico de la miel de abeja, mientras que el contenido de cenizas es un criterio de calidad para evaluar el origen botánico de la miel, que actualmente suele reemplazarse por la conductividad eléctrica (Bogdanov et al., 2018). De acuerdo con lo mencionado por Ruoff y Bogdanov, métodos como el análisis de polen, la determinación de humedad, el contenido de HMF, la medición de la actividad de invertasadiastasa, la composición de los azúcares, la conductividad eléctrica y el contenido de prolina se llevan a cabo como análisis de rutina de la calidad de la miel de abeja, no obstante es necesario 
contar con otros métodos como la espectroscopía infrarroja para realizar mediciones rápidas (Ruoff Kaspar, 2004). Según se muestra en el estudio "Exploratory Monitoring of the Quality and Authenticity of Commercial Honey in Ecuador" (Salvador, et al., 2019) en el que se analizaron 25 muestras comerciales provenientes de las provincias de Pichincha y Loja, la combinación de análisis físicoquímico con técnicas espectroscópicas vibracionales (Raman e IR) combinadas con métodos quimométricos como el Análisis de Componentes Principales (PCA) permiten una mejor diferenciación e interpretación de la determinación de la autenticidad del producto. Por ejemplo, en dicho estudio se mostró claramente, mediante el uso de las mencionadas técnicas, la diferencia significativa de dos de las 25 muestras analizadas, estas presentaron un alto porcentaje de sacarosa (23.3 \% y $38.91 \%$ ) y bajo porcentaje de azúcares reductores (53.3 \% y $38.42 \%$ ) en relación con los límites establecidos en la normativa vigente. Es necesario considerar la presencia de contaminantes en la miel de abeja, ya que, por ejemplo, un estudio realizado recientemente en los Laboratorios de AGROCALIDAD, tuvo como resultado que el $52 \%$ de las muestras de los apiarios analizados presenta residuos de oxitetraciclina (Vásquez, 2017), mientras que en el estudio "Exploratory Monitoring of the Quality and Authenticity of Commercial Honey in Ecuador" (Salvador, 2019) se analizó la presencia de 34 compuestos entre principios activos y metabolitos de pesticidas, y se detectó la presencia de DMF en una muestra, sustancia que es un metabolito del Amitraz (acaricida utilizado para controlar la varroosis), lo que indica que se están utilizando pesticidas en el Ecuador.

\section{Conclusiones y recomendaciones}

Los resultados de este estudio permiten contar con datos de los análisis físicoquímicos de 27 muestras de miel de abeja de Apis mellifera expendida como tal en los mercados de Quito y una muestra de miel artificial. Las muestras analizadas presentan un alto nivel de cumplimiento de los parámetros de la normativa vigente en el momento del estudio, excepto en una muestra que no cumple el parámetro de densidad y tres muestras que no cumplen el porcentaje de azúcares reductores. Además de comparar los resultados de la miel artificial con la miel de abeja.

En los sitios de expendio de los mercados del sur de Quito se encontró que se ofertan cinco marcas de miel de abeja, dos de las cuales no tienen registro sanitario, lo que no es indicativo de incumplimiento de la normativa, considerando que al ser la miel de abeja un producto natural no es obligatorio contar con registro sanitario (ahora notificación sanitaria) según lo mencionado en el artículo 14, literal b) de la Resolución ARCSA -DE-067-2015-GGG.

Al comparar los resultados de los análisis realizados en la miel artificial, se muestra que los valores varían en los siguientes parámetros: contenido de azúcares, sacarosa, diastasa, HMF y sólidos insolubles. Mientras que los resultados obtenidos en los parámetros restantes (densidad relativa, humedad, acidez total) es similar. Debido a que el contenido de HMF y de diastasa pueden deberse al sobrecalentamiento y almacenamiento prolongado, y que el contenido de sólidos insolubles es un indicador de higiene en el proceso, no pueden considerarse parámetros que permiten determinar la autenticidad del producto, siendo la cantidad y calidad de los azúcares el parámetro crítico, cuya determinación se puede realizar por diferentes técnicas analíticas. Sin embargo, es importante contar con análisis sencillos que permitan discriminar la miel genuina de la miel adulterada como el método del dietil éter o espectroscópicos (IR, Raman).

Al momento del estudio la norma INEN 1572 vigente fue la del año 1989, que fue actualizada en el año 2016. La nueva norma, en cuanto a parámetros de la miel CLASE I (para consumo hu- 
mano directo), presenta los siguientes cambios: la eliminación del parámetro de densidad relativa a $27^{\circ} \mathrm{C}$, la inclusión de valores diferenciados de sólidos insolubles en agua para miel distinta a la prensada (menor a $0.1 \%$ y miel prensada (menor a $0.5 \%$ ), el incremento de la acidez libre a máximo 50 meq/1000 g, el cambio de la actividad diastasa de 3 como mínimo y 8 como máximo, y la inclusión del parámetro de conductividad, por lo que se recomienda realizar estudios que evalúen la calidad de la miel basándose en los parámetros de esta norma vigente actualmente.

Finalmente, se recomienda realizar estudios de este tipo para contar con datos sobre la calidad de la miel de abeja producida en diferentes regiones del Ecuador, que aportaran para conocer cómo afectan algunos factores, entre ellos: la ubicación geográfica, la altitud, los tipos de floración, etc. Y cualquier otro estudio que aporte a conocer el potencial apícola en las diferentes zonas del país. Se sugiere además realizar estudios con técnicas como infrarrojo (IR) y espectroscopía de $1 \mathrm{H}-\mathrm{RMN}$ que permitirán conocer de mejor manera el producto que se comercializa en el país, así como también se muestra la necesidad de estudiar la presencia de contaminantes químicos y microbiológicos en este alimento, por su impacto en la salud humana y porque además son un factor que incide en la reducción de la población de abejas, insectos muy útiles y valiosos para el ser humano y para el ecosistema.

\section{Bibliografía}

AOAC. (1984). Oficial Methods of Análisis. Associations of Oficial Analytical Chemists. Fourteenth Edition. Virginia: Edited by Sydney Williams, Arlington.

ARCSA - MSP. (2015). RESOLUCIÓN ARCSA-DE-067-2015-GGG. Recuperado de https://www.controlsanitario. gob.ec/wp-content/uploads/downloads/2015/12/Resolucion_ARCSA-DE-067-2015-GGG.pdf

Bogdanov, S., et al. (30 de octubre de 2018). Calidad de la miel de abejas y estándares de control: Revisión realizada por la Comisión Internacional de la Miel. Recuperado de https://www.apiservices.biz/ documents/articulos-es/calidad_miel_de_abejas_y_estandares_de_control.pdf

CODEX STAN 12-2001. (s. f.). Obtenido de Codex Alimentarius: file:///C:/Users/Lorena/Downloads/ CXs_012s\%20(1).pdf

FAOSTAT. (2016). FAOSTAT. (FAO) Recuperado el 11/11/2017, de http://www.fao.org/faostat/es/\#data/QL

INEN. (1989). NTE INEN 1634. Miel de abejas. Determinación de la acidez total. Recuperado de http://181.112.149.204/buzon/normas/1634-C.pdf

INEN. (1989). NTE INEN 1636. Miel de abejas. Determinación del contenido de cenizas. Recuperado de http://181.112.149.204/buzon/normas/1636-C.pdf

INEN. (2012). NTE INEN 1635. Miel de abejas. Determinación del contenido de sólidos insolubles. Recuperado de INEN (1989). http://181.112.149.204/buzon/normas/1635-C.pdf

INEN. (2012). NTE INEN 1637. Miel de abejas. Determinación del contenido de hidroxi metil furfural.

INEN. (2016). NTE INEN 1572: 2016-10. Miel de abeja. Requisitos. Quito: INEN.

INEN. (1988). NTE INEN 1572: 2008-04 Miel de Abeja. Requisitos. Quito: INEN.

INEN. (2012). NORMA INEN 1632. Miel de abejas. Determinación de la densidad relativa a $27^{\circ} \mathrm{C}$ y de la humedad. Recuperado de. http://181.112.149.204/buzon/normas/1632-C.pdf

INVIMA. (23/03/ 2010). Resolución 1057. Recuperado de Requisitos sanitarios de la miel de abeja de consumo humano: file:///C:/Users/Lorena/Downloads/Res_1057_de_2010_Miel_de_abejas.pdf

Kirk, R. S. (1999). Composición y análisis de los alimentos. CDMX, Mexico: Continental.

Lorena Salvador, M. G. (2019). Exploratory Monitoring of the Quality and Authenticity of Commercial Honey in Ecuador. Foods.

MAG. (25/07/2015). Ecuador tiene potencial para la apicultura. Obtenido de Noticias del Ministerio de Agricultura y Ganadería: https://www.agricultura.gob.ec/ecuador-tiene-potencial-para-la-apicultura/ 
Martínez, W. (2009). Comparación de los parámetros de calidad en base a la Norma. Quito: Universidad Central del Ecuador.

Morales, R. C. (2016). Determinación de los parámetros físico- químicos para evaluar la calidad de la miel de abejas comercializada en la ciudad de Cuenca, según norma NTE INEN 1572 1998-04. Recuperado de http://dspace.uazuay.edu.ec/bitstream/datos/5469/1/11810.pdf

Noori Al-Waili, K. S.-G. (2012). Antibiotic, Pesticide, and Microbial Contaminants of Honey: Human Health Hazards. The Scientific World Journal, Article ID 930849, 9 pages.

Ruoff Kaspar, B. S. (2004). Authenticity of honey and other bee products. APIACTA 38, 317-327.

Vásquez, N. (Octubre de 2017). Repositorio de la Universidad Central del Ecuador. Obtenido de Determinación de Oxitetraciclina en muestra de miel de abeja: http://www.dspace.uce.edu.ec/ bitstream/25000/13311/1/T-UCE-0014-046-2017.pdf

Velásquez Giraldo A. V.; Zuloaga R. (2013). Physicochemical and Microbiological Characterization of Apis mellifera sp. Honey from Southwest of Antioquia in Colombia. Ingeniería y Ciencia, 9(18), 61-74.

Vit, P., Chievano, E., Zuccato, V., y Finotello, C. (2015). Authenticity of Ecuadorian Commercial Honeys. International Journal of Nutrition and Food Engineering, vol. 9(3), 327-330.

White, J. (1994). The Role of HMF and Diastase Assays in Honey Quality Evaluation. Bee World, 75(3), 104117. 